\title{
Influence of internal orifice geometry on synthetic jet performance
}

\author{
Girish K. Jankee ${ }^{1}$ B Bharathram Ganapathisubramani ${ }^{1}$
}

Received: 31 October 2018 / Revised: 7 February 2019 / Accepted: 11 February 2019 / Published online: 1 March 2019

(c) The Author(s) 2019

\begin{abstract}
The periodic ingestion and ejection of fluid through an orifice yield vortex rings forming an unsteady jet. The ability to impart momentum with a zero net mass flux makes synthetic jet actuators coveted components in flow control applications. Previous studies underlined the influence of geometrical parameters on synthetic jet evolution. This investigation introduces the orifice lip ratio as a new geometric variation that can influence the performance of the jet. We assess the sensitivity of synthetic jet efficiency to this parameter. Comparisons of test configurations establish the lip ratio as a pivotal parameter and indicate noticeable differences in the exit velocity. The results also highlight the dependence of the jet formation criterion on the orifice lip ratio. The analysis conclusively demonstrates the significance of the orifice lip ratio in the design and optimisation of synthetic jet actuators.
\end{abstract}

\section{Introduction}

The field of active flow control has experienced unprecedented growth over the past decades, largely attributed to the expanding knowledge of fluid mechanics as well as the advent of novel control strategies (Cattafesta and Sheplak 2011). This investigation focuses on synthetic jet actuators (SJAs) and discusses the importance of the orifice internal geometry in enhancing the mean blowing velocity of the jet.

In the field of flow control, the momentum flux at the orifice exit and the energy consumption of the driver characterise the efficiency of the actuator. Performance enhancement can be achieved by optimising the fluidic properties, driver properties and actuator geometry, as categorised by Feero et al. (2015). Ugrina and Flatau (2004) and Holman et al. (2005) found the orifice diameter, the nozzle length, the radius of curvature of the outer orifice edge and cavity volume to be key geometrical aspects affecting the performance of the actuator. Gallas et al. (2003) found that the actuator response depends on the relative position of the cavity and diaphragm resonance while Glezer and Amitay

Girish K. Jankee

G.Jankee@soton.ac.uk

Bharathram Ganapathisubramani

G.Bharath@soton.ac.uk

1 Aerodynamics and Flight Mechanics, Faculty of Engineering and Physical Sciences, University of Southampton,

Southampton SO17 1BJ, UK
(2002) and Chaudhari et al. (2009) improved the effectiveness by ensuring the diaphragm and cavity are driven at a coupled resonance.

Utturkar et al. (2002) performed incompressible flow simulations on rectangular cavities and concluded that the actuator response is independent of cavity shape and diaphragm placement. However, Gallas (2005) observed the flow to be compressible for $f / f_{h}>0.5$, where $f_{h}$ is the cavity resonance frequency and this contributes to fluctuations in fluid temperature in the orifice. Hence, there are shortcomings associated with incompressible flow simulations. Van Buren et al. (2016) also experienced compressibility effects while modifying the disc properties and architecture. For a doubled displaced volume, the velocity of the jet did not double. Instead, only a $40 \%$ increase was noted. At high velocities, compressibility effects become increasingly important and the temperature difference has to be accounted for. A higher temperature difference was measured for the dual disc configuration implying that part of the energy heats up the flow rather than increasing the velocity.

Van Buren et al. (2015) and Feero et al. (2015) also focused on cavity shapes. The former showed that pan-cake shaped cavities contain acoustic resonances that are inaccurately predicted using Helmholtz resonance theory, thus undermining the concept of lumped element modelling (LEM). Feero et al. (2015) compared a cylindrical, a conical and a contraction-shaped cavity for an axisymmetric orifice. The investigation concluded that due to a lower cavity 
resonance frequency, a more complex shape (contraction) consumes less power and achieves highest energy efficiency when operated at resonance. Maximum momentum flux was achieved by the cylindrical-shaped cavity which prompted the authors to hypothesise that vortices which get ingested into the cavity during suction contribute to blockage of the flow for complex geometries, leading to poor performance. The rectangular shaped cavity provides enough space to accommodate the ingested vortices and this results in higher exit velocities.

Orifice properties also show interesting bearings in influencing the flow characteristics. Nani and Smith (2012) found that the acoustic power dissipation, as a result of synthetic jet formation, is strongly dependent on the radius of the inner orifice lip. Rounding the inner orifice lip reduced the acoustic power by more than $40 \%$ until the orifice channel no longer possessed a straight portion. This appeared to inhibit flow reattachment on the inward walls. Gomes and Crowther (2006) found that the boundary layer thickness increases with channel length due to the shear action of the slow fluid moving near the wall and the fast-moving flow in the core. Such behaviour has the tendency to reduce the peak velocity of the synthetic jet.

Although various studies have contemplated actuator geometry as a means to improve the performance, there still remains a recurring issue for bidirectional flow control devices such as synthetic jet actuators. Due to the oscillatory behaviour of the flow, necking occurs in long orifice channel configurations. Ward-Smith (1979) investigated the height of the orifice channel and characterised the existence of a threshold aspect ratio (AR) value $\left(h_{0} / d_{0}=0.75\right)$ beyond which flow reattachment occurs (Fig. 1). Since synthetic jets are bidirectional, during the suction stroke, the flow separates at the outer orifice edges and a free shear layer grows within the orifice channel. If the channel is long enough (high AR), the flow reattaches to the orifice channel walls and an oscillating separation bubble gets trapped. This reduces the effective orifice diameter and
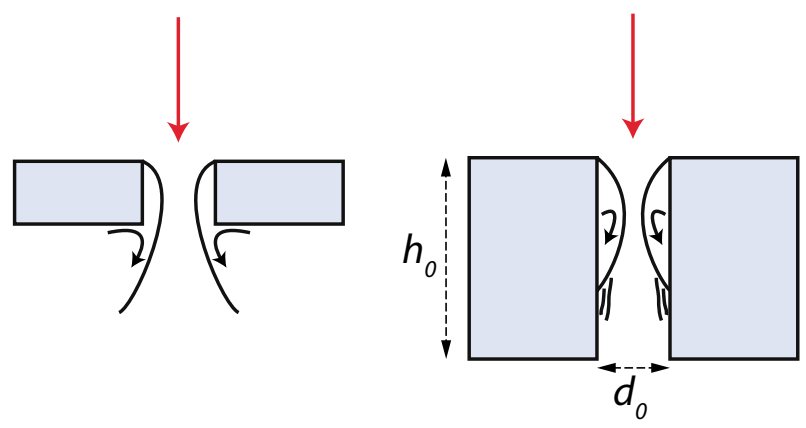

Fig. 1 Schematic showing the problematic influence of necking for high AR SJAs (adapted from Ward-Smith (1979)). The AR is defined as $h_{0} / d_{0}$, where $h_{0}$ is the height of the orifice channel and $d_{0}$ is the orifice diameter increases the stiffness of the system. Necking leads to blockage of the flow during subsequent blowing phases culminating in poor performance displayed by the actuator.

The aim of the present study is to tackle the problem of necking for high AR orifices by introducing an orifice inner lip, which arises from previous investigations by Ward-Smith (1979) and Gomes and Crowther (2006). The primary objective is to quantify the sensitivity of the synthetic jet performance on this singular parameter. Secondly, this paper attempts to establish the influence of the orifice lip dimension on synthetic jet formation (Fig. 2).

\section{Governing parameters}

While describing the behaviour of synthetic jets, some assumptions need to be made. Firstly, it is inferred that the velocity of the jet is constant over the exit plane due to the simple top-hat velocity profile at the orifice exit. Although this overestimates the spatial velocity and the total mass flow at most locations of the jet exit (Feero et al. 2015), measurements performed by Smith and Glezer (2005) and Aydemir et al. (2012) support the above approximation and show that the jet centerline velocity at the orifice exit follows a sinusoidal profile, given by

$u_{0}(t)=u_{\max } \sin \left(\frac{2 \pi t}{T}\right)$,

where $u_{\max }(\mathrm{m} / \mathrm{s}), t(\mathrm{~s})$ and $T(\mathrm{~s})$ represents the maximum velocity during the blowing cycle, the time and the period of the cycle, respectively. Further, following Smith and Glezer (1998), the mean blowing velocity, $\bar{u}$, is used as a representative velocity throughout and is defined as

$\bar{u}=\frac{1}{T} \int_{0}^{T / 2} u_{0}(t) \mathrm{d} t=\frac{u_{\max }}{\pi}$.

The synthetic jet flowfield also depends on a displacement amplitude, viz. the stroke length, $L_{0}(\mathrm{~m})$, which is based on a slug model where it represents the length of the slug of fluid ejected during each cycle. The dimensionless stroke length, $L$, is given by

$L=\frac{L_{0}}{d_{0}}=\frac{\bar{u}}{f d_{0}}$,

where $f(\mathrm{~Hz})$ is the actuation frequency of the synthetic jet.

In addition, Glezer and Amitay (2002) characterised the synthetic jet by the Reynolds number. In this study, it depends on the orifice diameter, $d_{0}(\mathrm{~m})$, and the mean blowing velocity, $\bar{u}(\mathrm{~m} / \mathrm{s})$, while the viscous effects are described by the kinematic viscosity, $v\left(\mathrm{~m}^{2} / \mathrm{s}\right)$. The actuation frequency can be expressed as the non-dimensional Stokes number, $S$. 


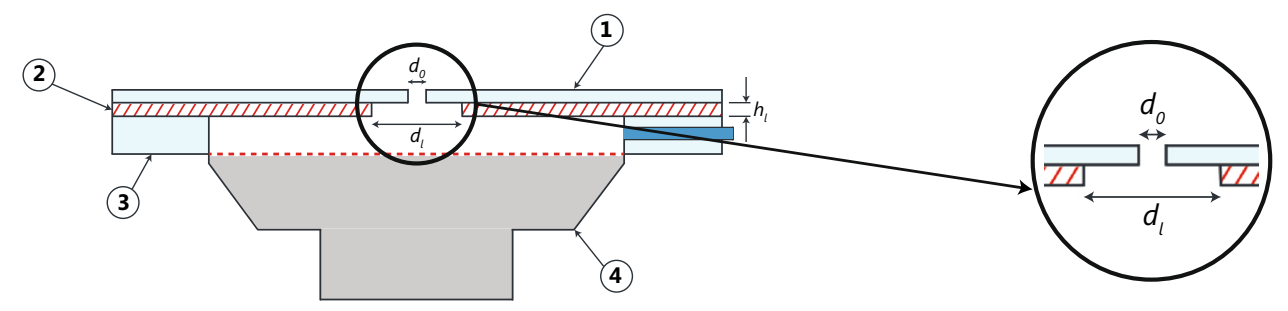

Fig. 2 Cross-section of SJA with focus on the orifice lip ratio. In the current configuration, an orifice exit plate and an orifice inner lip plate make up the orifice assembly. (1. Orifice exit plate 2. Orifice inner lip plate 3. Cavity assembly 4. Loudspeaker)

$\operatorname{Re}=\frac{\bar{u} d_{0}}{v}$

$S=\sqrt{\frac{2 \pi f d_{0}^{2}}{v}}$.

Vortex formation is dictated by circulation which increases up to the point where pinch-off occurs. Pinch-off is the moment at which circulation stops flowing into the vortex sheet and it breaks away to form a vortex ring which advects away under its self-induced velocity. Experimental data from Smith et al. (1999) and Shuster and Smith (2004) showed a pinch-off threshold of $L_{0} / d_{0}$ equivalent to 1 for axisymmetric orifices. Results from Rosenfeld et al. (1998) and O'Farrell and Dabiri (2014) corroborated these findings to establish the universality of this formation number for axisymmetric orifices. Further, Holman et al. (2005) established the concept of threshold criterion for the formation of synthetic jets for axisymmetric and rectangular orifices. A jet is bound to form if

$\frac{R e}{S^{2}}>C$,

For axisymmetric orifices, the constant is 0.16 . This empirically determined value was found to be consistent with data from Smith et al. (1999).

\section{Experimental setup}

This simple table-top experiment makes use of a synthetic jet actuator (SJA), a pressure transducer, constant temperature anemometry and particle image velocimetry.

\subsection{Actuator design}

The SJA consists of three components: the driver, the cavity assembly and the orifice assembly (Fig. 3). The loudspeaker was attached to the cavity assembly and the orifice, both milled from aluminium plates. The same cavity assembly, with a volume, $V_{c}$, of $2.63056 \times 10^{-5} \mathrm{~m}^{3}$, was used for all test cases. The axisymmetric orifice has a diameter, $d_{0}$, of $7.05 \mathrm{~mm}$ resulting in a cross-sectional area, $A_{0}$, of $39 \mathrm{~mm}^{2}$. The SJA is driven by a Visaton SC $8 \mathrm{~N}$ speaker. The loudspeaker has an impedance of $8 \Omega$ with a rated power of $30 \mathrm{~W}$ and a frequency response between 70 and $20,000 \mathrm{~Hz}$, with diaphragm resonance of around $110 \mathrm{~Hz}$. An in-house code allows control of the speaker by sending a sinusoidal signal (Eq. 7) from a data acquisition (DAQ) device to the loudspeaker via an amplifier. It is important to apply a gain to the signal as the DAQ device can only handle up to a maximum of $10 \mathrm{~V}$.

$V_{\text {out }}=A \sin \left(2 \pi f t_{s}\right)$,

where, $V_{\text {out }}(\mathrm{V})$ is the output signal from the DAQ device to the amplifier, $A$ is the amplitude of the signal prior to amplification, $f(\mathrm{~Hz})$ is the actuation frequency of the loudspeaker and $t_{s}(\mathrm{~s})$ is the duration over which the signal is sampled. The amplitude and frequency of the signal can be adjusted within the in-house code to alter the operating conditions.

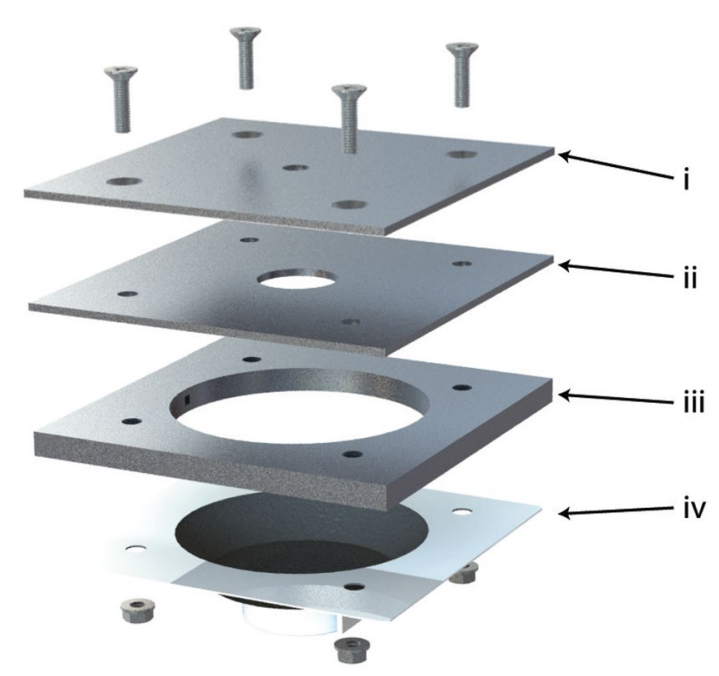

Fig. 3 Exploded assembly schematic of SJA. i. Orifice exit plate. ii. Orifice lip plate. iii. Cavity assembly. iv. Loudspeaker 


\subsection{Constant temperature anemometry}

Velocity measurements in the orifice exit plane were performed using hot-wire anemometry. A single-wire traversing hot-wire probe with diameter, $d_{w}$, of $2.5 \mu \mathrm{m}$ and prong spacing of $2.5 \mathrm{~mm}$ was operated in constant temperature mode with an overheat ratio of 0.8 . The wire resistance was measured to be $5 \Omega$. The probe was aligned such that the sensing bit was positioned at the center of the orifice exit (Fig. 4). An anemometer modular rack system was used to measure the voltage variation of the hot-wire probe. Calibration was performed pre- and postdata acquisition, using King's law, to compensate for any drift in temperature. Using a two-axis traverse, hot-wire measurements were performed at a wall normal distance of $6 \mathrm{~d}_{0}$ away from the orifice exit. Post processing involved removing rectification of the mean blowing velocity by assuming that the larger peak in the cycle corresponded to the blowing phase. Such conjecture is valid provided the velocity is measured downstream of the exit plane as the magnitude of the ingestion velocity decreases rapidly while moving away from the orifice exit (Feero et al. 2015).

\subsection{Pressure transducer}

A pressure transducer was fitted inside the cavity to monitor the variation in pressure during the blowing and the suction phases. An Endevco 8507C-2 pressure transducer, with a diameter of $2.5 \mathrm{~mm}$, was mounted flushed with the inner walls of the cavity (Fig. 4). To acquire measurements, an NI-PXIe 1062Q data acquisition computer is fitted with an NI-PXIe 4330 card for bridge based measurements. By subjecting the pressure transducer to a known frequency and amplitude signal in a control environment, its sensitivity was found to be $117.1 \mathrm{mV} / \mathrm{PSI}$. Background

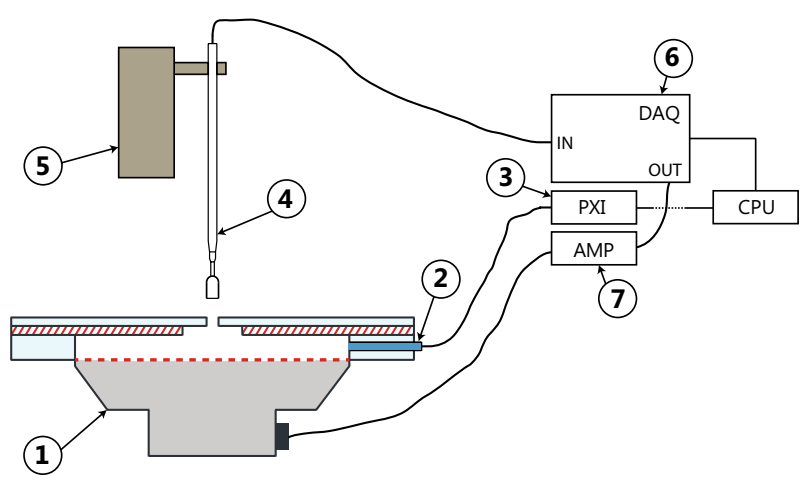

Fig. 4 Experimental setup showing constant temperature anemometry device and a pressure transducer fitted inside the cavity. (1. SJA 2. Pressure transducer 3. NI PXIe-4330 4. Probe holder and hot wire sensor 5. 2-axis traverse system 6. NI USB-6251 7. Amplifier)

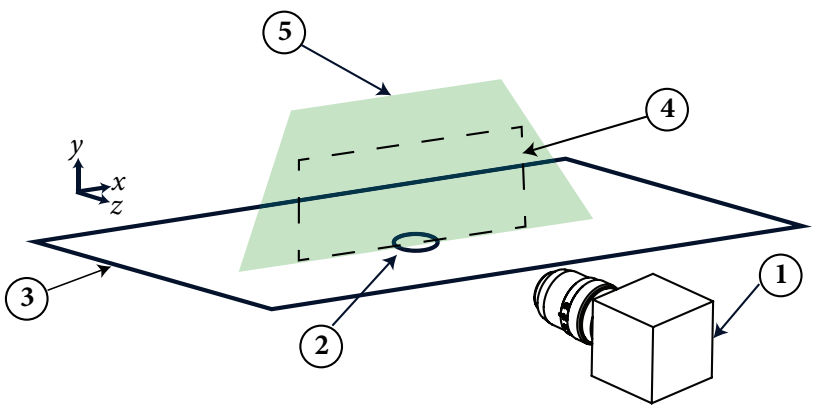

Fig. 5 Sketch of planar PIV setup.(1. Camera and lens assembly 2. Orifice exit 3. Test section 4. Region of interest 5. Laser sheet)

noise was initially recorded and subtracted from measurements during post-processing. Data from the pressure transducer and the hot-wire anemometry were acquired simultaneously.

\subsection{Planar PIV}

Phase-locked planar PIV measurements were taken in the $\mathrm{xy}$-plane, where $x$ and $y$ correspond to the spanwise and wall-normal streamwise direction, respectively (Fig. 5). A Litron $200 \mathrm{~mJ}$ dual-pulse Nd-YAG system was used to create a single laser sheet with a thickness of approximately $1 \mathrm{~mm}$. The laser sheet was positioned along the centerline of the orifice. Seeding is provided by a Martin Magnum 1200 smoke machine, ejecting smoke particles with a mean diameter of $1 \mu \mathrm{m}$ into a closed-test section.

A LaVision Imager Pro LX 16MP camera, fitted with a $200 \mathrm{~mm}$ focal length lens, was used to capture image pairs with a delay of $20 \mu$ s between the frames. The laser and the camera were phase-locked to the driving signal of the actuator. For each of the 8 phases, 200 image pairs were recorded. Vector fields were determined using LaVision Davis 8.3.0 software. A first step with a window size of $48 \times 48$ pixels, followed by two steps of $24 \times 24$ pixels with $75 \%$ overlap were applied, resulting in a resolution of one vector per $0.166 \mathrm{~mm}$. The measurement plane spans from $-5 d_{0}$ to $5 d_{0}$ in the spanwise direction and up to $15 d_{0}$ in the wall-normal direction.

\subsection{Test cases}

This paper defines the orifice lip ratio as a novel means of altering the internal geometry of the orifice. Measurements of the pressure variation within the cavity confirm that a change in lip ratio does not alter the cavity properties. A deviation of less than $7 \%$ in mean blowing cavity pressure, considered inconsequential, is observed with a 
Table 1 The combination of lip ratios and AR resulted in 12 differing test configurations

\begin{tabular}{ll}
\hline & Axisymmetric orifice \\
\hline AR & $0.57,0.85$ \\
$\mathrm{R}_{l}$ & $1,1.5,2,3,5,9$ \\
\hline
\end{tabular}

change in total volume of the assembly as the lip ratio varies. Further analysis of the data shows that there is less than 5\% variation between frequency values at which cavity resonance occurs for differing orifice lip test cases. This emphasises the assumption that this study is targeted specifically at the orifice geometry while the cavity properties remain unchanged. In the current configuration, the orifice lip ratio, $R_{1}$, represents the ratio of the inner lip diameter, $d_{1}$, and the orifice exit diameter, $d_{0}$ (Eq. 8). Variation of this unique parameter results in a number of test cases (Table 1).

$R_{1}=\frac{d_{1}}{d_{0}}$

In an attempt to eliminate any possible influence of the make or condition of the speaker, the results are presented with respect to a baseline configuration. The case with a lip ratio, $R_{1}$, of 9 is taken as the baseline case as it is conformed to widely used geometrical specifications where the AR is low. This normalisation procedure also allows repeatability of the experiment under contrasting actuation mechanisms. Further, unless otherwise specified, the results correspond to the same amplitude of the input signal as defined in Eq. 7.

\section{Results}

\subsection{Necking effects}

From the frequency response of the actuator, it is possible to isolate the effects of the orifice channel height. A degradation in the performance of the actuator is observed with an increase in AR as the flow encounters more resistance. There exists a damping effect on the flow curvature and an increase in boundary layer thickness since the fluid moves faster in the core as compared to closer to the walls, due to the presence of shear forces. This leads to a reduction in the effective diameter of the orifice and the problem of necking arises which weakens the actuator's performance (Fig. 6).

Further evidence of necking is provided by pressure measurements within the cavity. A reduced-order model developed by Persoons and O'Donovan (2007) is used to relate the average velocity at the orifice exit to the cavity

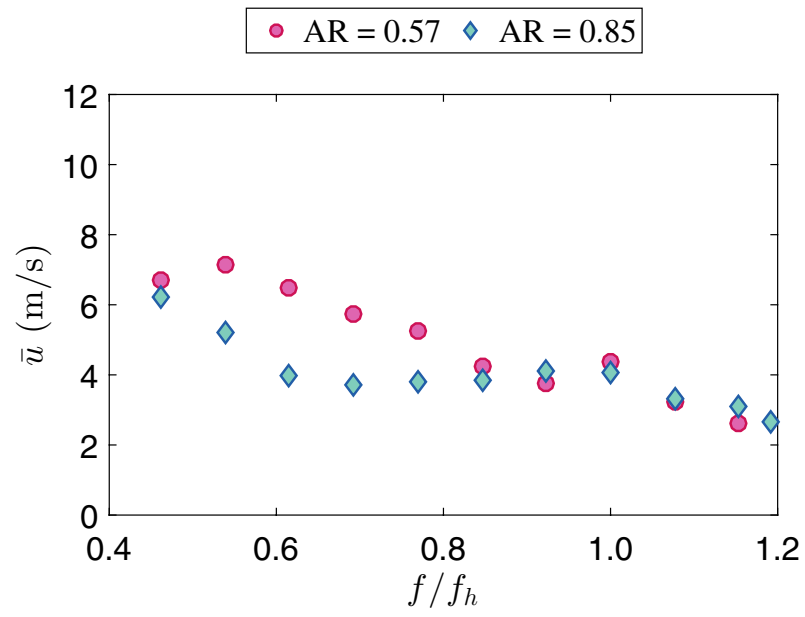

Fig. 6 The plot compares the influence of the orifice channel height (AR) on the exit velocity of the actuator. The input amplitude is kept fixed while the actuation frequency is varied

pressure. The use of pressure-velocity characterisation is often favoured since voltage-velocity characterisation depends on the driver properties and cannot account for actuator degradation or other external influence. For known cavity pressure, geometric parameters and fluid parameters of synthetic jets, the following equation is applied.

$\bar{u}=\frac{\overline{p^{\prime}}}{\rho c} \sqrt{\frac{2 V_{c}}{A_{0} L_{n}^{\prime}}} \frac{1}{\sqrt{\left(\frac{f}{f_{h}}\right)^{2}+\sqrt{\left(\frac{f}{f_{h}}\right)^{4}+\kappa^{2}\left(\frac{V_{c} \bar{p}^{\prime}}{A_{0} L_{n}^{\prime} \rho c^{2}}\right)^{2}}}}$,

with $L_{n}^{\prime}$ given by

$L_{n}^{\prime}=L_{n}+2 \beta \sqrt{\frac{4 A_{0}}{\pi}}$,

where $\bar{p}$ is the mean pressure within the cavity over the blowing phase, $\kappa$ is a non-linear damping loss coefficient and $\beta$ is an added mass coefficient for the orifice.

There exists a large overestimation of the exit velocity from the pressure transducer data for the case with $R_{l}$ of 1 . Variations over $55 \%$ can be observed (Fig. 7a). Such discrepancy can be attributed to the blockage effects generated within the orifice channel, which lead to an increase in back pressure within the cavity. This clogging effect likely contributes to a slower jet and leads to inaccuracies in the pressure-velocity characterisation. These results highlight the limitations of Persoon's model for high AR orifice channels where necking is increasingly prevalent. 


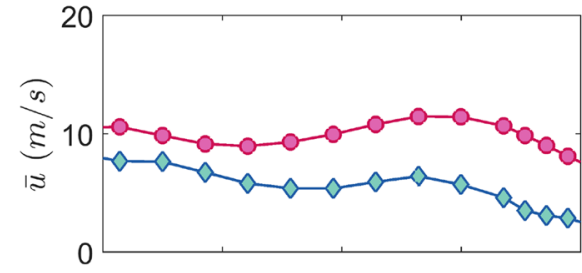

(a) $R_{l}=1$

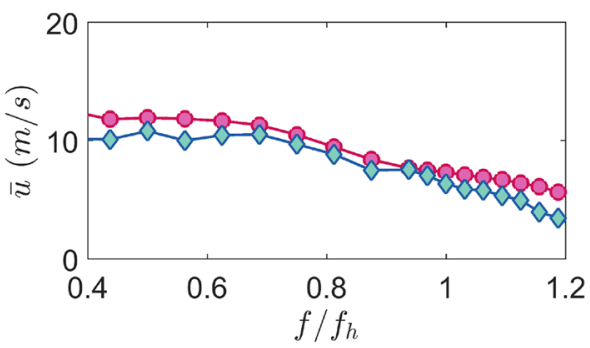

(d) $R_{l}=3$
Pressure transducer $\diamond-$ Hot wire

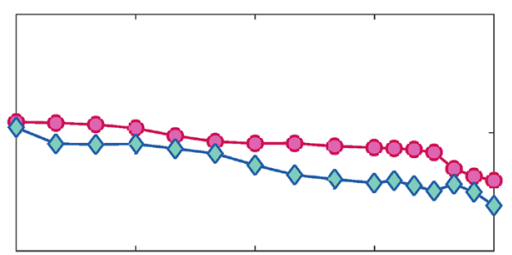

(b) $R_{l}=1.5$

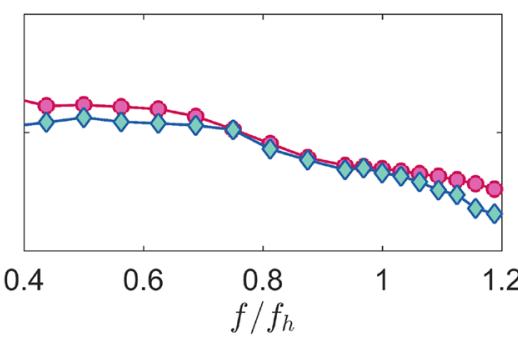

(e) $R_{l}=5$

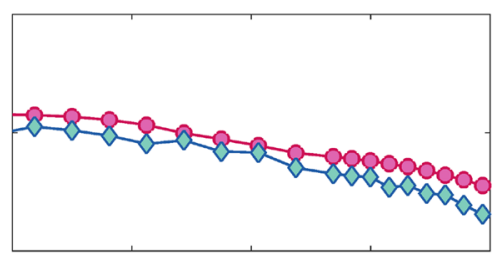

(c) $R_{l}=2$

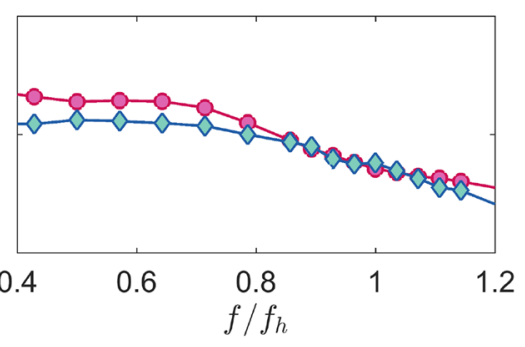

(f) $R_{l}=9$

Fig. 7 Comparison between hot-wire and pressure-based jet exit velocity. Discrepancy between values provides evidence of necking effects while an increase in lip ratio mitigates the inconsistency in the datasets

\subsection{Orifice lip ratio}

To counter the influence of necking, this study assesses the impact of inner orifice lips on the flowfield of SJAs. Inspection of Fig. 8 indicates a noticeable influence of the lip ratio in high Stokes number regimes. Two key features emerge from the analysis. Firstly, the presence of a lip minimises the heterogeneous behaviour between low and high AR test cases. Secondly, there exists an ideal lip size beyond which any increase in inner lip diameter does not result in any further enhancement in performance. Further, the inconsistency between exit velocity values derived from pressure transducer and hot-wire measurements diminishes as the lip ratio increases, implying that the influence of necking can be forestalled by the presence of an inner orifice lip (Fig. 7).

Such trend occurs due to the bidirectional nature of synthetic jet actuators. During the suction phase, a free shear layer develops within the orifice and vortex roll-up occurs along the walls of the channel. The presence of inner lips provides enough space to accommodate these structures and mitigates their contribution to a reduction in effective orifice diameter. An alleviation in necking effects inhibits blockage of the flow during subsequent blowing phases, culminating in higher mean blowing velocity achieved at the orifice exit. An optimum lip ratio exists because a vortex ring can only have a finite amount of vorticity and hence, its diameter is limited. Once the vortex ring with maximum diameter can be accommodated, any ensuing increase in lip ratio has no impact on ameliorating the output. From the experimental

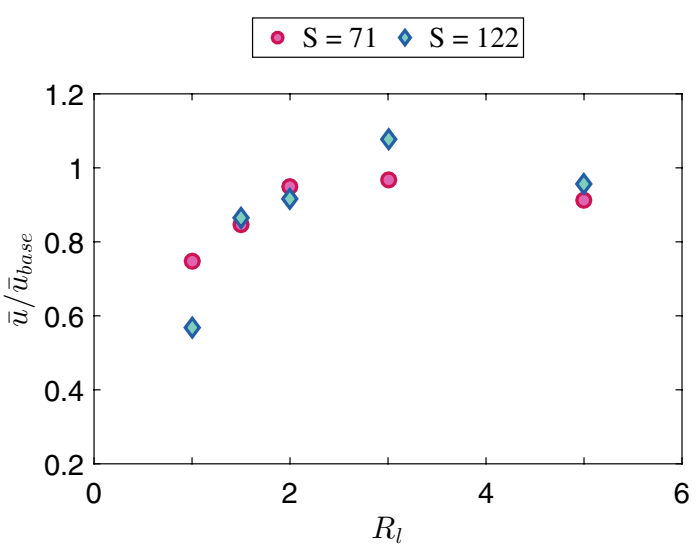

Fig. 8 Plot of the variation of the normalised mean blowing velocity with lip ratio for two different Stokes number cases at $A R=0.85$. The mean blowing velocity of the baseline configuration $\left(R_{1}=9\right)$ is used as a normalising factor

data, the exit velocity varies minimally between lip ratios of 3 and 9 . Hence, the threshold for lip ratio for the current configuration is $R_{1}=3$.

A change in lip ratio can also be perceived as commensurate to a modification in the degree of contraction of the orifice channel, especially at high Stokes number. Jain et al. (2011) investigated the degree of convergence on the orifice performance by simulating a bevel-shaped orifice with a varying angle. The results showed that an increase in angle reduced the peak exit velocity since the fluid encountered 
more resistance by travelling a longer distance along the orifice channel. It was concluded that, for bidirectional actuators such as synthetic jets, convergence is useful only during the blowing phase and can entrain a potentially deleterious outcome during suction. Using parallels from Jain et al. (2011), the presence of inner orifice lips introduces a mechanism similar to virtual aeroshaping by allowing convergence to occur naturally within the orifice channel (Fig. 9). During suction, accommodation of the flow structures generated fills up the void created by the inner lips but shedding of vorticity at the sharp lip edges during subsequent blowing engenders a degree of curvature on the outgoing flow which possibly experiences an acceleration. Consequently, inner lips provide an upgrade for fixed wall convergence in the case of bidirectional flows as it tackles necking during suction whilst accelerating the flow during blowing.

\subsection{Vortex pinch-off criterion}

Using an order of magnitude analysis, simulations and experiments, Holman et al. (2005) found a formation criterion for axisymmetric orifice synthetic jet actuators of approximately $R e / S^{2} \approx 0.16$. Smith and Swift (2001) defined jet formation as the condition when the ejected vortex pair advects far enough not to experience the influence of suction. The dimensionless stroke length and spatial and temporal point of pinch-off provide an indication of jet formation. As circulation stops flowing into the vortex ring, it detaches and moves away under its self-induced velocity. Provided the impulse is high enough or the Stokes number is sufficiently low, jet formation can be achieved. Smith et al. (1999) showed that for an axisymmetric orifice, a jet is formed if $L_{0} / d_{0}$ is greater than 1 . Below this threshold, a vortex ring may form but pinch-off does not ensues. The

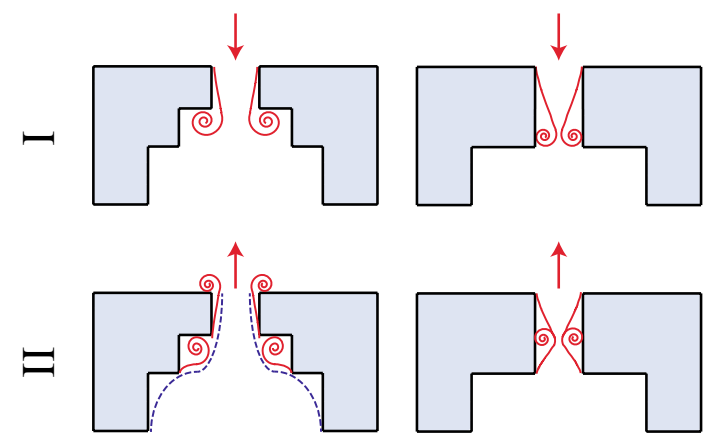

Fig. 9 Sketch of the hypothesised flow phenomena occurring within the orifice channel. I. During suction, the presence of inner lips provides enough space to accommodate these structures while for the case of $R_{1}=1$, reattachment of the flow occurs. II. During the subsequent blowing phase, a recirculation region is formed in the dead region due to vortex shedding at the sharp lip edges which leads to a virtual contraction, contributing to accelerating the flow. For $R_{1}=1$, blockage of the flow occurs exact value of this constant can potentially change depending on the geometricalproperties of the actuator, and therefore, pre-defined threshold values may not be universal.

Figure 10 assesses the impact of the orifice lip ratio on vortex formation based on the definition of Holman et al. (2005). A distinct trend emerges from the scattering of the datasets. Under the same operating conditions, a jet can be more easily synthesised if the actuator configuration comprises a lip ratio. An increase in lip ratio favours jet formation and vortex pinch-off. Since the formation criterion is characterised by the stroke length, actuator configurations which generate a larger slug of fluid during blowing are more likely to generate a jet. Following prior explanation, as the blockage effects diminish with an increase in orifice lip ratio, the jet exit velocity is enhanced. Subsequently, the dimensionless stroke length increases and lies above the threshold value. Hence, altering the internal geometry of the orifice by introducing an inner lip can effectively extend the range over which the vortex ring can be generated.

A peculiar case occurs at a Stokes number of 122 (Fig. 10). Actuator configuration with $R_{1}=1$ is below the pre-defined threshold value while $R_{1}=9$ lies above it. This implies that at that specific operating condition, $R_{1}=9$ is expected to generate vortices and synthesise a jet while $R_{1}=1$ does not meet the necessary conditions for jet formation. Analysis of the synthetic jet spectra can provide substantial understanding of the expected flow phenomena. From Fig. 11, unique traits can be observed. In the case of $R_{1}=9$, the driving frequency is denoted by the highest energy peak and is followed by smaller intensity peaks (subharmonics). This also represents the frequency at which vortex shedding occurs and sub-harmonics are representative of the various cycles during which pinch-off takes place. In the case of $R_{1}=1$, the spectra has a lower energy content and the absence of any peaks or harmonics confirms that vortex

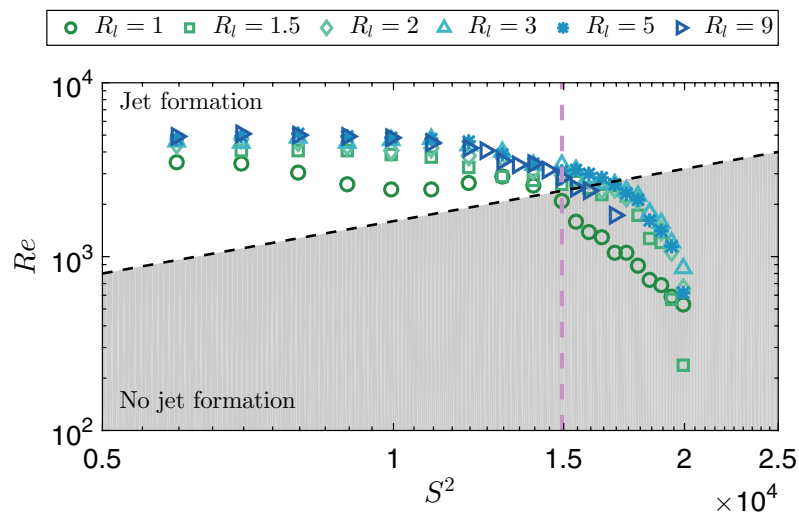

Fig. 10 Jet formation criterion based on Holman's definition with a threshold value of 0.16 for axisymmetric orifices (Holman et al. 2005). The pink dotted line denotes a Stokes number of 122 


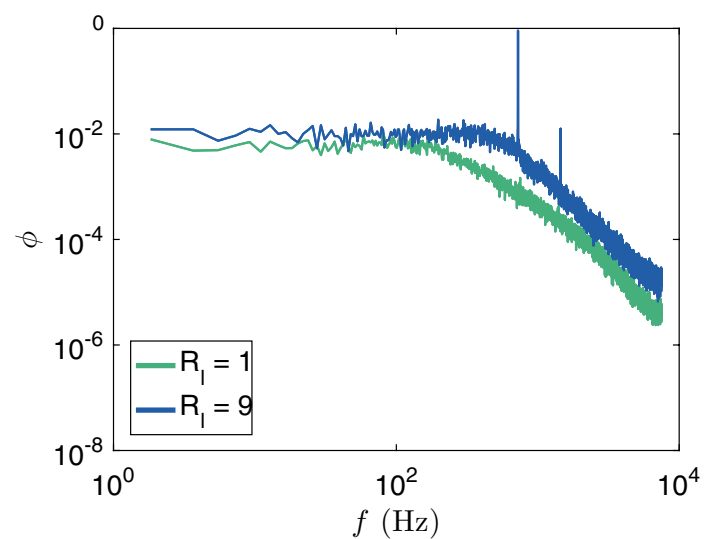

Fig. 11 Energy spectra based on hot-wire signal at a Stokes number of 122 . The high energy peak and corresponding harmonics correspond to vortex pinch-off pinch-off does not occur. Further, this validates the threshold values denoted by Smith et al. (1999) and Holman et al. (2005) and advocates their universality for axisymmetricshaped orifices.

Additional evidence of the influence of orifice inner lips on vortex pinch-off is provided by the planar PIV data. Figure 12 demonstrates that in the case of a lip ratio, vortex pinch-off occurs. During blowing $\left(\phi=0^{\circ}\right.$ and $\left.90^{\circ}\right)$, the vortex sheet rolls into a pair of well-defined vortex rings which pinches-off and advects away from the orifice during the suction phase $\left(\phi=180^{\circ}\right.$ and $\left.270^{\circ}\right)$. For the high AR orifice channel, vortex roll-up does not occur. It is likely that the vortex roll-up process is disrupted by the influence of necking. Instead, it can be observed that a faint trail of vorticity is shed throughout the cycle. Sharp edges engender more effective shedding of vorticity. Hence, it can be inferred that
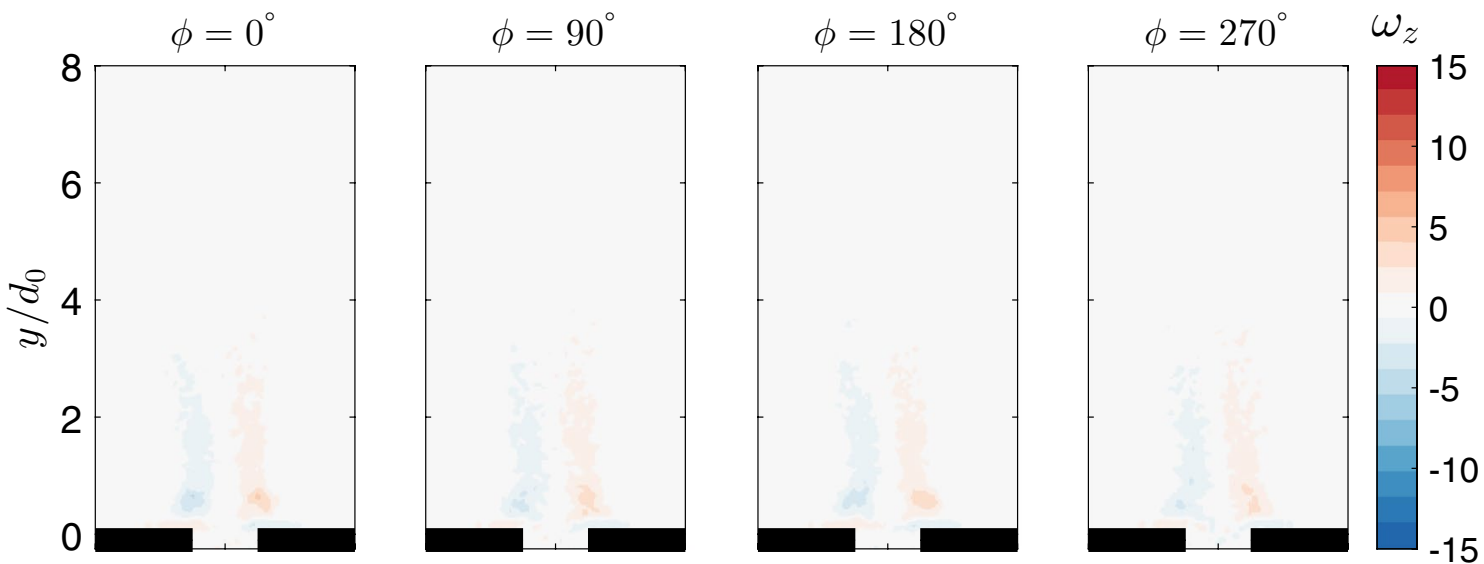

(a) $R_{l}=1$
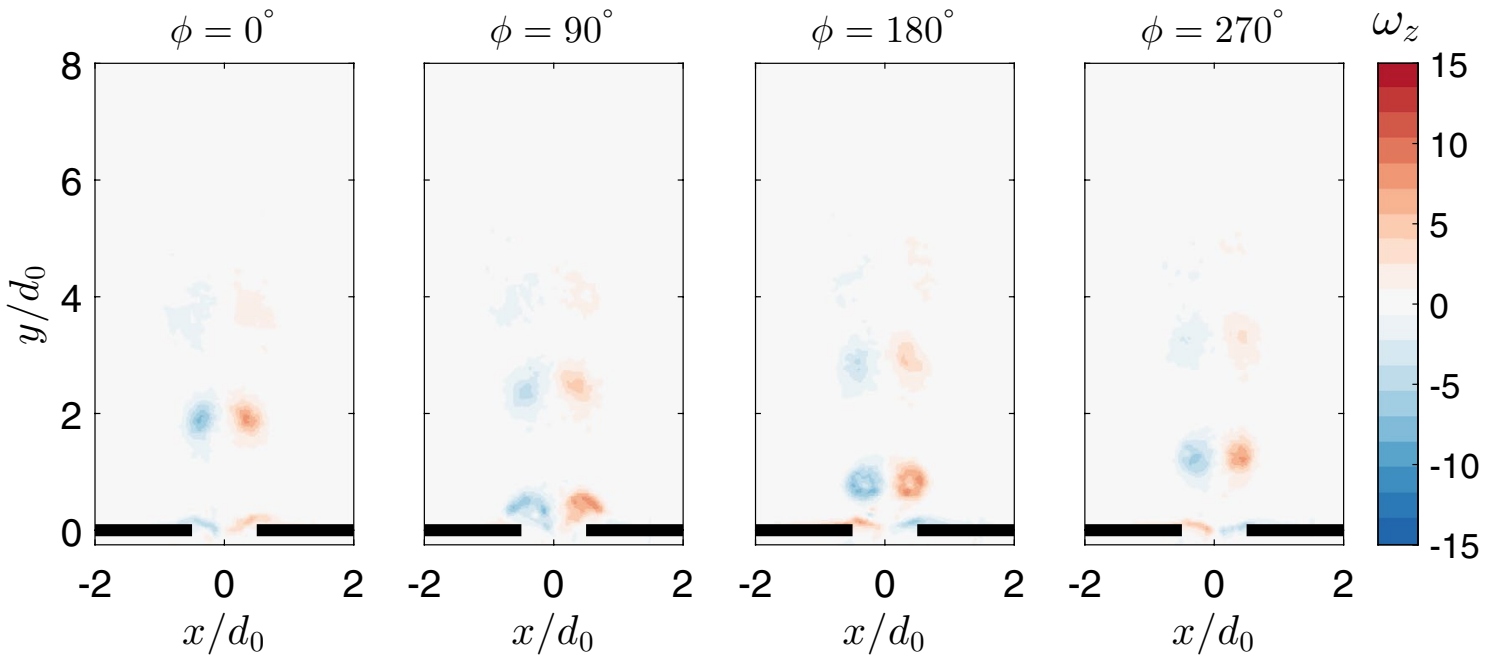

(b) $R_{l}=9$

Fig. 12 Phase-locked vorticity maps, at a Stokes number of 122, illustrating the case of a no vortex pinch-off for $R_{1}=1$ and b vortex pinch-off for $R_{1}=9$. Red background indicates positive vorticity while blue background indicates negative vorticity. A phase of $90^{\circ}$ corresponds to peak blowing while a phase of $180^{\circ}$ corresponds to peak suction 
the larger the number of sharp edges within the orifice channel, the more pronounced is the vortex shedding process. Based on the direct influence of inner orifice lips and its consequential by-products observed in this experiment, it is possible to devise a protocol which allows the design of more effective orifices for bidirectional flows.

From the literature, a global AR can be defined as the ratio of the total height of the channel and the orifice exit diameter $\left(h_{\mathrm{t}} / d_{0}\right)$. Even though this global AR does not comply with the threshold criterion of 0.75 for the flow not to reattach with the walls, provided the local AR of each inner lip is within the limit defined by Ward-Smith (1979), there is an enhancement in the amount of vorticity shed and jet exit velocity. From Fig. 13, this can be mathematically expressed using Eqs. 11-13.

The height of the orifice channel can be expressed as a summation of the smaller lip heights.

$h_{\mathrm{t}}=h_{0}+\sum_{i=1}^{n} h_{i}$

The threshold for flow reattachment during suction is an aspect ratio of 0.75 . Hence provided that locally

$\frac{h_{n}}{d_{n}}<0.75$

there will be an improvement in jet exit velocity even though $h_{\mathrm{t}} / d_{0}$ is greater than 0.75 . Further, Fig. 8 indicates the ideal lip ratio size to counter the influence of necking

$\frac{d_{n+1}}{d_{n}} \geq 3$

A combination of the above equations is proposed as an adequate method to design the internal geometry of the orifice, if the aim is to maximise the mean blowing velocity of a SJA. Such an approach can lead to the design of a new style of actuator, potentially suited for a number of applications, not limited to aerodynamics. SJA are primarily used since operating them at resonant frequencies provides a surge in the jet exit velocity. An actuator with inner lips will experience a further boost in exit velocity at resonant frequencies which involve auxiliary repercussions. For instance, the ability to achieve vortex pinch-off at a lower input voltage not only renders a SJA fitted with an orifice lip more efficient, but it also improves the mixing attributes of the device when operated at cavity resonance. Such characteristics are of particular interest where synthetic jets are used for impingement cooling over heated surfaces in smallscale electronic devices such as circuit board components. The range of Stokes number used in such applications being similar to the one tested in this study (Garg et al. 2005; Arik 2008), deploying our actuator under such conditions would
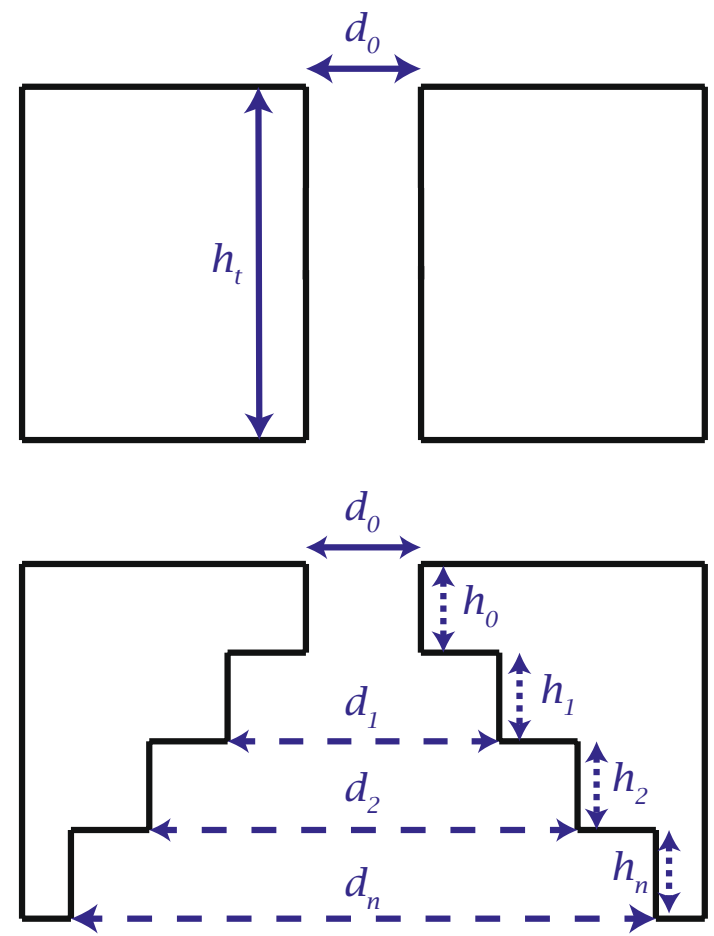

Fig. 13 Schematic of the proposed internal orifice geometry design for synthetic jet actuators based on experimental results

lead to enhanced mixing and heat transfer, but at a lower operational cost.

\section{Conclusion}

An experimental investigation was carried out to tackle necking in high AR orifices. The influence of the internal orifice geometry on such a problem was studied by introducing a novel concept defined as the orifice lip ratio. Simple table-top experiments were carried out using constant temperature anemometry, coupled with a pressure transducer placed within the cavity for data acquisition.

The severity of necking was determined by isolating the effects of the orifice channel height and examining the discrepancy between voltage and pressure-based velocity characterisation. Addition of an inner orifice lip led to an improvement in behaviour for high AR orifices. Such trend was observed since the inner lips provided enough space to accommodate the flow structures formed within the orifice channel during the suction stroke which mitigated their contribution to a reduction in effective orifice diameter. This led to an increase in jet momentum being observed. Moreover, the presence of an optimum lip ratio was recorded as the vortex ring can only have a limited amount of vorticity which restricts the maximum possible diameter of the ring. 
Jet formation and vortex pinch-off were also found to be sensitive to the orifice lip ratio. Configurations with inner lips achieved a larger stroke length and met the pre-requisites for jet formation to take place. Such behaviour was confirmed by the energy spectra where a high energy peak and corresponding harmonics denoted vortex pinch-off for actuator configurations bearing a lip ratio. Likewise, vorticity maps from planar PIV data also corroborated the features of the energy spectra and showed well-defined vortex pairs being synthesised when the orifice is fitted with an inner lip. The results also confirmed the universality of the threshold values determined by Holman et al. (2005) and Smith et al. (1999) for axisymmetric orifices.

In contrast to studies which examined complex actuator geometry modifications (Feero et al. 2015; Gomes and Crowther 2006; Van Buren et al. 2016), this research showed that a simple change in the orifice geometry improves jet exit velocity. More importantly, the orifice lip ratio can effectively ameliorate the performance of a synthetic jet actuator, while at the same time extending the range of operating conditions over which a jet can be synthesised.

Acknowledgements The authors acknowledge the financial support from the Engineering and Physical Sciences Research Council (ESPRC Grant No. EP/L006383/1) and Airbus. All data supporting this study are openly available from the University of Southampton repository at https://doi.org/10.5258/SOTON/D0813.

Open Access This article is distributed under the terms of the Creative Commons Attribution 4.0 International License (http://creativeco mmons.org/licenses/by/4.0/), which permits unrestricted use, distribution, and reproduction in any medium, provided you give appropriate credit to the original author(s) and the source, provide a link to the Creative Commons license, and indicate if changes were made.

\section{References}

Arik M (2008) Local heat transfer coefficients of a high-frequency synthetic jet during impingement cooling over flat surfaces. Heat Transf Eng 29(9):763-773. https://doi.org/10.1080/01457 630802053769

Aydemir E, Worth NA, Dawson JR (2012) The formation of vortex rings in a strongly forced round jet. Exp Fluids 52(3):729-742. https://doi.org/10.1007/s00348-011-1110-6

Cattafesta LN, Sheplak M (2011) Actuators for active flow control. Annu Rev Fluid Mech 43(1):247-272. https://doi.org/10.1146/ annurev-fluid-122109-160634

Chaudhari M, Verma G, Puranik B, Agrawal A (2009) Frequency response of a synthetic jet cavity. Exp Therm Fluid Sci 33(3):439-448. https://doi.org/10.1016/j.expthermfl usci.2008.10.008

Feero MA, Lavoie P, Sullivan PE (2015) Influence of cavity shape on synthetic jet performance. Sens Actuators A Phys 223:1-10. https://doi.org/10.1016/j.sna.2014.12.004

Gallas Q (2005) On the Modeling and Design of Zero-Net Mass Flux Actuators. PhD thesis, University of Florida
Gallas Q, Wang G, Papila M, Sheplak M, Cattafesta L (2003) Optimization of Synthetic Jet Actuators. In: 41st Aerospace Sciences Meeting and Exhibit, American Institute of Aeronautics and Astronautics, Reston, Virigina, Aerospace Sciences Meetings. https://doi.org/10.2514/6.2003-635

Garg J, Arik M, Weaver S (2005) Impingement air cooling With synthetic jets over small and large heated surfaces. In: Advanced Electronic Packaging Parts A, B, C, ASME, vol 2, pp 277-285. https://doi.org/10.1115/IPACK2005-73211

Glezer A, Amitay M (2002) Synthetic Jets. Annu Rev Fluid Mech 34(1):503-529. https://doi.org/10.1146/annurev.fluid.34.09050 1.094913

Gomes L, Crowther W (2006) Towards a practical piezoceramic diaphragm based synthetic jet actuator for high subsonic applications - effect of chamber and orifice depth on actuator peak velocity. In: 3rd AIAA Flow Control Conference, American Institute of Aeronautics and Astronautics, Reston, Virigina, pp 1-17. https://doi.org/10.2514/6.2006-2859

Holman R, Utturkar Y, Mittal R, Smith BL, Cattafesta L (2005) Formation criterion for synthetic jets. AIAA J 43(10):2110-2116. https://doi.org/10.2514/1.12033

Jain M, Puranik B, Agrawal A (2011) A numerical investigation of effects of cavity and orifice parameters on the characteristics of a synthetic jet flow. Sens Actuators A Phys 165(2):351-366. https://doi.org/10.1016/j.sna.2010.11.001

Nani DJ, Smith BL (2012) Effect of orifice inner lip radius on synthetic jet efficiency. Phys Fluids 24(11):115110. https://doi. org/10.1063/1.4767725

O'Farrell C, Dabiri JO (2014) Pinch-off of non-axisymmetric vortex rings. J Fluid Mech 740:61-96. https://doi.org/10.1017/ jfm.2013.639

Persoons T, O'Donovan TS (2007) A pressure-based estimate of synthetic jet velocity. Phys Fluids 19(12):128104. https://doi. org/10.1063/1.2823560

Rosenfeld M, Rambod E, Gharib M (1998) Circulation and formation number of laminar vortex rings. J Fluid Mech 376:297-318. https://doi.org/10.1017/S0022112098003115

Shuster J, Smith D (2004) A Study of the Formation and Scaling of a Synthetic Jet. In: 42nd AIAA Aerospace Sciences Meeting and Exhibit, American Institute of Aeronautics and Astronautics, Reno, Nevada. https://doi.org/10.2514/6.2004-90

Smith B, Swift G (2001) Synthetic jets at large Reynolds number and comparison to continuous jets. In: 15th AIAA Computational Fluid Dynamics Conference, American Institute of Aeronautics and Astronautics, Reston, Virigina, vol 836, https://doi. org/10.2514/6.2001-3030

Smith B, Trautman M, Glezer A (1999) Controlled interactions of adjacent synthetic jets. In: 37 th Aerospace Sciences Meeting and Exhibit, American Institute of Aeronautics and Astronautics, Reno, Nevada, https://doi.org/10.2514/6.1999-669

Smith BL, Glezer A (1998) The formation and evolution of synthetic jets. Phys Fluids 10(9):2281-2297. https://doi. org/10.1063/1.869828

Smith BL, Glezer A (2005) Vectoring of adjacent synthetic jets. AIAA J 43(10):2117-2124. https://doi.org/10.2514/1.12910

Ugrina S, Flatau AB (2004) Investigation of synthetic jet actuator design parameters. In: Flatau AB (ed) Smart Materials and Structures, vol 5390, p 284. https://doi.org/10.1117/12.547541

Utturkar Y, Mittal R, Rampunggoon P, Cattafesta L (2002) Sensitivity of synthetic jets to the design of the jet cavity. In: 40th AIAA Aerospace Sciences Meetings, American Institute of Aeronautics and Astronautics, Reno, Nevada, pp 1-8. https:// doi.org/10.2514/6.2002-124

Van Buren T, Whalen E, Amitay M (2015) Synthetic jet actuator cavity acoustics: Helmholtz versus quarter-wave resonance. J Vib Acoust 137(5):054501. https://doi.org/10.1115/1.4030216 
Van Buren T, Whalen E, Amitay M (2016) Achieving a HighSpeed and Momentum Synthetic Jet Actuator. J Aerosp Eng 29(2):04015040. https://doi.org/10.1061/(ASCE)AS.19435525.0000530

Ward-Smith AJ (1979) Critical flowmetering: the characteristics of cylindrical nozzles with sharp upstream edges. Int J Heat
Fluid Flow 1(3):123-132. https://doi.org/10.1016/0142727X(79)90028-6

Publisher's Note Springer Nature remains neutral with regard to jurisdictional claims in published maps and institutional affiliations 